\title{
Methicillin-resistant Staphylococcus aureus enterocolitis sequentially complicated with septic arthritis: a case report and review of the literature
}

\author{
Yukari Ogawa ${ }^{1}$, Takeshi Saraya ${ }^{1 *}$, Takashi Koide ${ }^{1}$, Ken Kikuchi $^{2}$, Kosuke Ohkuma$^{1}$, Koji Araki $^{3}$, Hiroshi Makino ${ }^{3}$, \\ Shota Yonetani ${ }^{3}$, Hajime Takizawa' and Hajime Goto ${ }^{1}$
}

\begin{abstract}
Background: Although most reports describing patients infected with methicillin-resistant Staphylococcus aureus enterocolitis have been published in Japan, this concept remains a matter of debate and diagnostic criteria have not yet been defined.

Case presentation: The general status of a 74-year-old Japanese man referred to our hospital (day 1) with severe community-acquired pneumococcal pneumonia gradually improved with antibiotic therapy. Thereafter, up to 4 L/day of acute watery diarrhea that started on day 19 was refractory to metronidazole but responded immediately to oral vancomycin. Gram staining stool samples was positive for abundant fecal leukocytes from which dominant methicillin-resistant Staphylococcus aureus $\left(10^{4} \mathrm{CFU} / \mathrm{mL}\right)$ were isolated, suggesting methicillin-resistant Staphylococcus aureus enterocolitis. High fever with methicillin-resistant Staphylococcus aureus bacteremia was evident at day 30, and suppurative right hip arthritis developed around day 71. All methicillin-resistant Staphylococcus aureus strains isolated from stools, blood and aspirated synovial fluid separated in the same manner on pulsed-field gel electrophoresis, as well as two other strains isolated from sputum, belonged to the same clone as sequence type (ST) 764 (complex clonal 5), and carried SCCmec type II.

Conclusion: The clinical, microbiological and molecular biological findings of this patient indicated methicillinresistant Staphylococcus aureus enterocolitis that led to septic methicillin-resistant Staphylococcus aureus arthritis.
\end{abstract}

Keywords: Methicillin-resistant Staphylococcus aureus, Fecal leukocytes, Multilocus sequence typing

\section{Background}

We describe a 74-year-old man who was diagnosed with methicillin-resistant Staphylococcus aureus (MRSA) enterocolitis that subsequently caused septic arthritis. We reviewed reports of MRSA enterocolitis published in Japan over the past 10 years and characterized the clinical features of MRSA enterocolitis. Here, we discuss the clinical significance of staphylococcal enterotoxins (SEs) and toxic shock syndrome toxin-1 (TSST-1).

\footnotetext{
*Correspondence: sara@yd5.so-net.ne.jp

'Department of Respiratory Medicine, Kyorin University School of Medicine, 6-20-2 Shinkawa, Mitaka, Tokyo 181-8611, Japan

Full list of author information is available at the end of the article
}

\section{Case presentation}

A 74-year-old Japanese man was transferred to our department from a local hospital under a diagnosis of pneumonia. He had been in good health five days before transfer to our hospital, but with pyrexia and dyspnea upon effort. He had been treated with oral antihyperglycemic drugs to control type 2 diabetes mellitus five years previously. He was a carpenter who did not consume alcohol, but had a smoking history of 44 pack-years. Although he could walk unaided, he seemed very ill. His consciousness level was E4V5M6 (Glasgow coma scale), and his vital signs were: blood pressure, 90/50 mmHg; temperature, $37.3^{\circ} \mathrm{C}$; heart rate, 86 beats per min with sinus rhythm; respiratory rate, 24 breaths per minute; and oxygen saturation, $78 \%$ with an oxygen supply of $10 \mathrm{~L} / \mathrm{min}$ delivered via a reservoir mask. He was 
immediately admitted to the intensive care unit (ICU). Physical findings were normal except for coarse crackles in the bilateral middle to lower lung fields. Upon admission to our hospital (day 1), a chest X-ray revealed infiltration throughout all lung fields except the left upper lung. These results together with positive findings of pneumococcal urinary antigens indicated a diagnosis of pneumococcal pneumonia (CURB 65 score was 3, which is defined as Confusion, Urea nitrogen, Respiratory rate, Blood pressure, and 65 years of age and older; pneumonia severity index was class IV). He was immediately intubated and treated with intravenous meropenem (1.5 g/day) plus ciprofloxacin (600 mg/day), which gradually improved his respiratory status over a period of two weeks. However, acute watery diarrhea amounting to $4 \mathrm{~L} /$ day (determined by inserting a rectal tube) together with pyrexia $\left(38^{\circ} \mathrm{C}\right)$ was evident on day 19 (Figure 1). Although stool specimens were negative for Clostridium difficile toxins $\mathrm{A}$ and $\mathrm{B}$, pseudomembranous colitis was considered and metronidazole (1 g/day) was started via a nasogastric tube (Figure 1). However, the watery diarrhea was refractory to metronidazole, and Gram staining stool samples revealed abundant fecal leukocytes (Figure 2), suggesting colonic membranous damage. Although colonic fiberscopy on day 22 provided limited information only as far as the sigmoid portion because the health status of the patient was unstable, definitive evidence of pseudomembranous formation other than membranous edema or hyperemia was not found. Furthermore, methicillin-resistant Staphylococcus aureus (MRSA; $10^{4}$ colony forming unit/mL) was predominantly isolated from stool cultures on day 19 whereas other enteric bacterial pathogens and parasites or ova were negative, indicating MRSA enterocolitis. Vancomycin (VCM; 1 g/day) delivered via a nasogastric tube completely resolved the watery diarrhea within a few days. However, at day 30, high fever recurred with moderate watery diarrhea $(\sim 500 \mathrm{~mL} /$ day $)$, and one of two sets of blood cultures was positive for MRSA. Thereafter, intravenous VCM (0.5 g/day) with dose adjustment for renal dysfunction was initiated, and repeated blood cultures were negative for MRSA. The total amount of watery diarrhea excreted between days 19 and 34 was $22.43 \mathrm{~L}$ and the maximum volume per $24 \mathrm{~h}$ reached $3.50 \mathrm{~L}$ on day 25.

The general status of the patient gradually improved except for persistent low-grade fever, and he was able to walk unaided by day 64 . Intermittent pain in the right hip joint started on day 71 after he started rehabilitation for walking (Figure 1). A physical examination revealed the obturator or psoas sign, and he was positive for the Gaenslen maneuver, suggesting inflammation at the retroperitoneal or lumbar vertebrae and/or sacroiliac joint. We explored the source of the fever and right hip joint pain using magnetic resonance imaging (MRI) (Figure $3 \mathrm{~A}$ and $\mathrm{B}$ ) and ${ }^{67}$ gallium scintigraphy of the hip

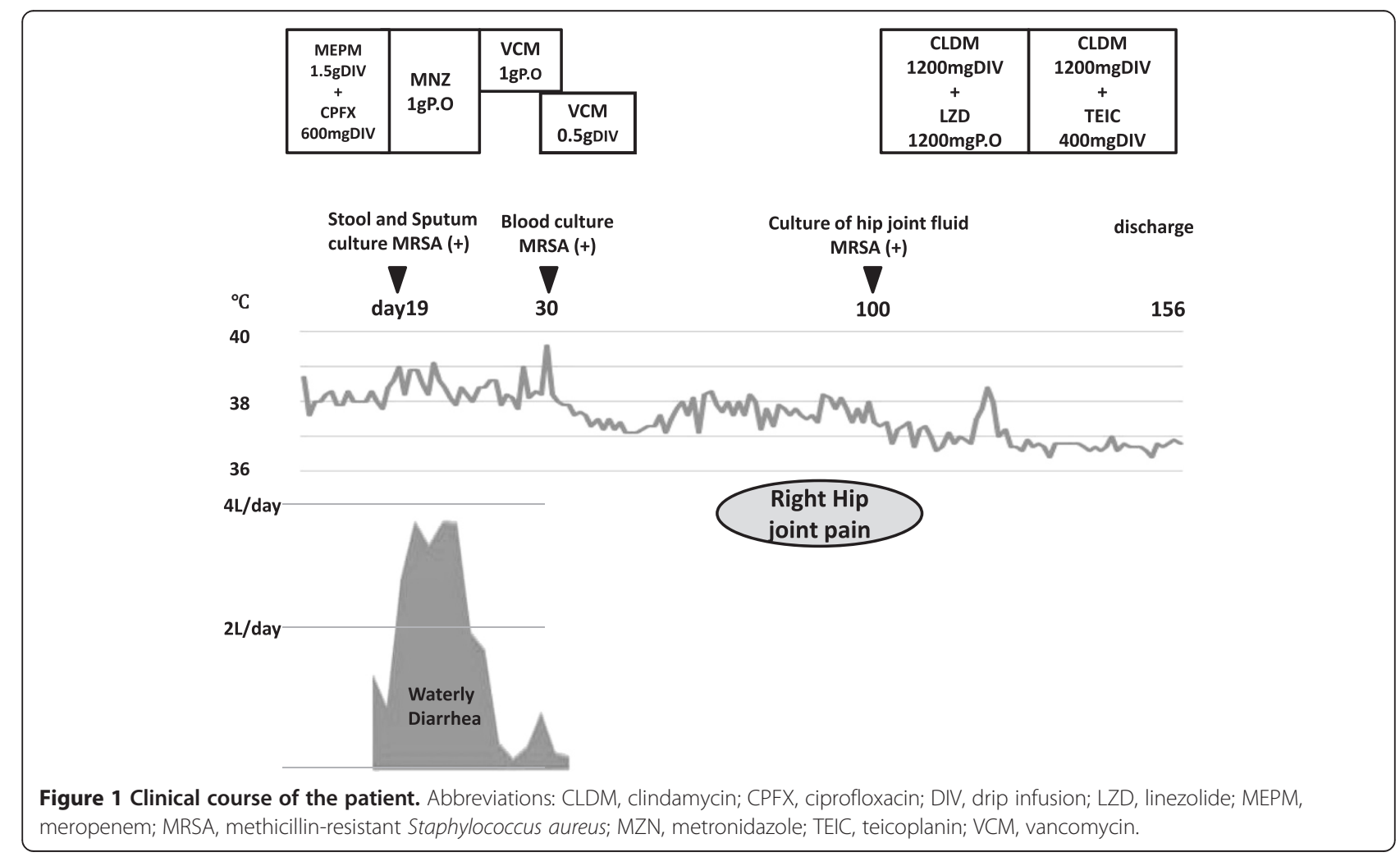




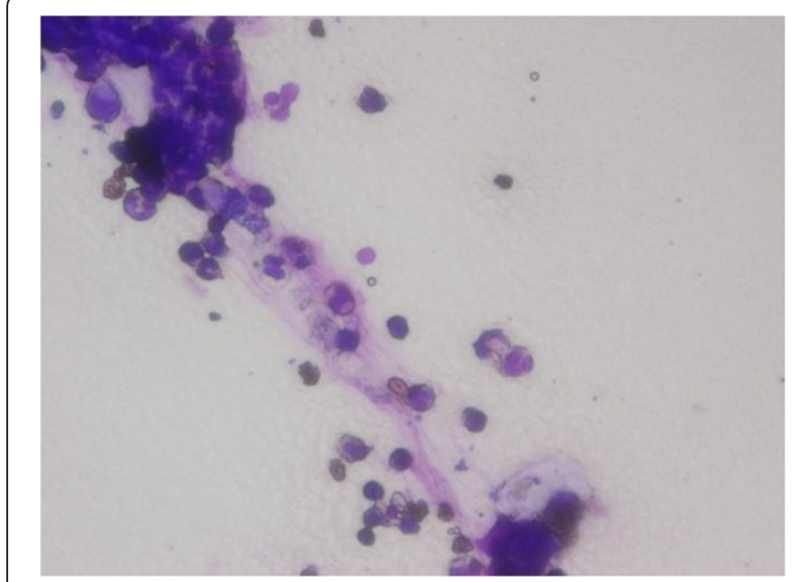

Figure 2 Gram staining findings of stool cultures. Fecal leukocytes are abundant.

joint. T2-weighted MRI (T2WI) revealed an area of high intensity around the head of the right femur indicating fluid collection (Figure 3A). Fat-saturated T2-weighted images (T2WI) revealed another area of high intensity on the right obturator externus or adducent muscles (Figure 3B), suggesting right hip arthritis that ${ }^{67}$ gallium scintigraphy confirmed as a hotspot (Figure 3C). Joint drainage fluid collected on day 100 showed abundant leukocytes enclosed by phagocytic gram-positive cocci (Figure 3D) that were later identified as MRSA. Pulsed- field gel electrophoresis (PFGE) showed that the MRSA strains in the samples isolated from stool (day 19), blood (day 30) and synovial fluid (day 100) were identical (Figure 4), suggesting a hematological spread of MRSA originating from the colonic membrane damaged by MRSA enterocolitis. The PFGE profiles of two strains isolated from sputum on days 10 and 44 and that of a strain isolated from a catheter tip on day 20 were similar, but all three strains differed by one band from strains isolated from stool, blood and synovial fluid specimens. These strains were considered to be derived from the same clone with different subtypes according to Tenover's criteria [1], and they had the same antibiogram $(\mathrm{VCM} \leq 1$, teicoplanin $\leq 1$, linezolide 2$)$. These findings suggested that persistent colonized MRSA could coevolve even in a host environment.

None of the findings were consistent with the criteria for confirmed toxic shock syndrome (TSS; fever $>38.9^{\circ} \mathrm{C}$, hypotension $\leq 90 \mathrm{mmHg}$ ), diffuse erythroderma, desquamation (unless death occurs before desquamation) and the involvement of at least three organ systems) or probable TSS (missing one characteristic for confirmed TSS) [2] or pseudomembranous colitis. Thus, he was finally diagnosed with MRSA enterocolitis that subsequently caused septic arthritis in the right hip.

Indeed, multilocus sequence typing confirmed that the allelic profiles of the MRSA strains obtained from blood, sputum and synovial fluid on days 30,45 and 100 ,

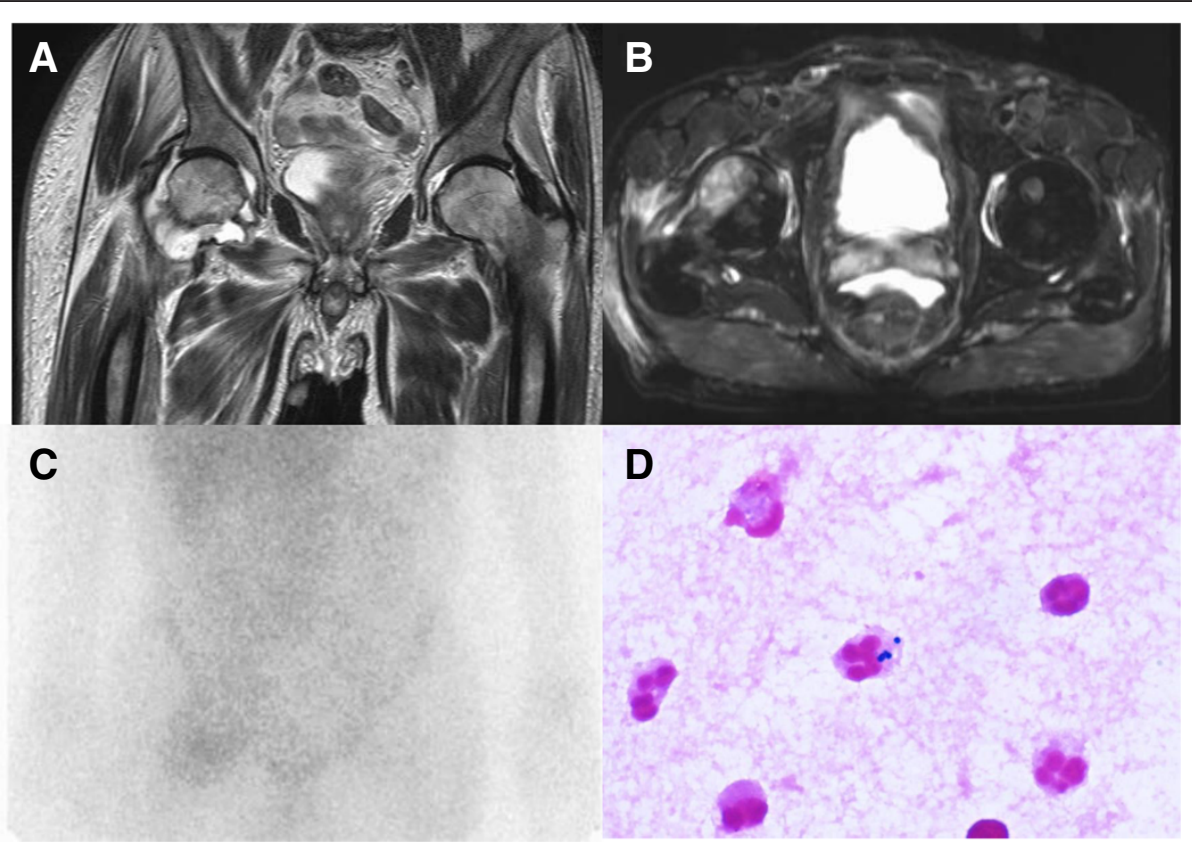

Figure 3 Magnetic resonance imaging of right hip joint. T2-weighted image (T2WI) of hip joint shows high-intensity area surrounding head of right femur, indicating fluid collection (A). Fat saturated T2WI shows high-intensity area on right obturator externus or adducent muscles (B) that appears as a hotspot on gallium scintigram (C). Synovial fluid contains abundant leukocytes that are phagocytically engulfed by grampositive cocci (D) 


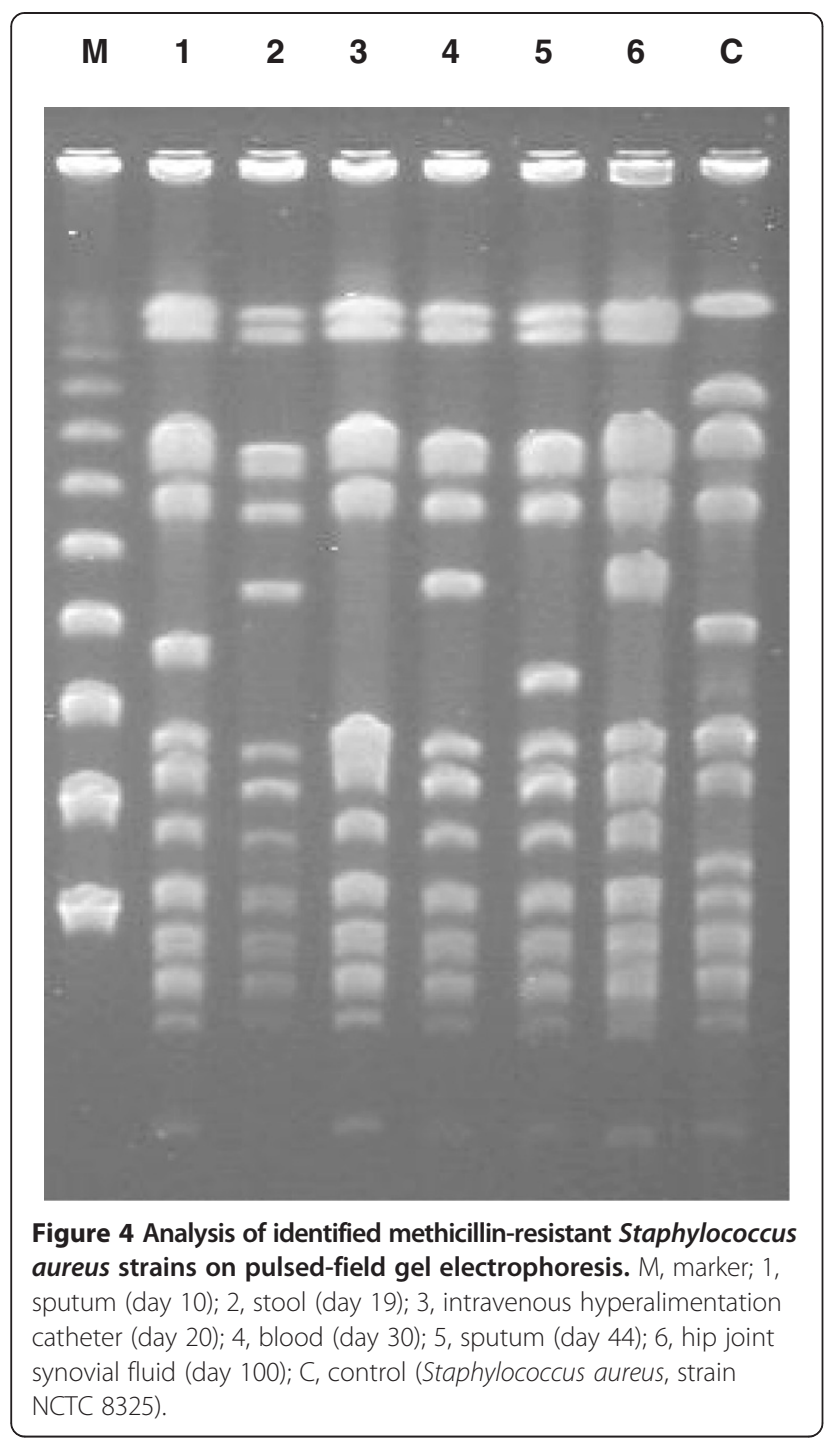

Table 1 Toxin gene profiles and multilocus sequence typing of MRSA strains obtained from blood, sputum, and synovial fluid

\begin{tabular}{|c|c|c|c|c|c|c|c|c|c|c|c|c|c|c|c|c|c|c|c|c|c|c|c|c|c|}
\hline \multirow[t]{2}{*}{ Source } & \multicolumn{13}{|c|}{ Detection of toxic genes } & \multicolumn{3}{|c|}{ scCmec } & \multicolumn{9}{|c|}{ MLST } \\
\hline & tst & eta & etb & sea & seb & $\sec$ & sed & see & seg & seh & sei & sej & $p v l$ & $\begin{array}{l}\text { mec } \\
\text { type }\end{array}$ & $\begin{array}{c}c c r \\
\text { type }\end{array}$ & $\begin{array}{l}\mathrm{SCC} \\
\mathrm{mec}\end{array}$ & $\operatorname{arcC}$ & aroE & $g l p F$ & $g m k$ & pta & tpi & yqil & ST & $C C$ \\
\hline A. blood & - & - & - & - & - & - & - & - & + & - & + & - & - & A & 2 & $\|$ & 1 & 136 & 1 & 4 & 12 & 1 & 10 & 764 & 5 \\
\hline Sputum & - & - & - & - & - & - & - & - & + & - & + & - & - & A & 2 & $\|$ & 1 & 136 & 1 & 4 & 12 & 1 & 10 & 764 & 5 \\
\hline Joint fluid & - & - & - & - & - & - & - & - & + & - & + & - & - & A & 2 & $\|$ & 1 & 136 & 1 & 4 & 12 & 1 & 10 & 764 & 5 \\
\hline
\end{tabular}

A: acetyltransferase, arc: carbamate kinase, aro: shikimate dehydrogenase, CC: clonal complex, ccr: cassette chromosome recombinase, eta: exfoliatin toxin a etb: exfoliatin toxin b, glp: glycerol kinase, gmk: guanylate kinase, MLST: multilocus sequence typing, pta: phosphate acetyltransferase, pvl: Panton-Valentine leukocidine, sea: staphylococcal enterotoxin a, seb: staphylococcal enterotoxin b, sec: staphylococcal enterotoxin c, sed: staphylococcal enterotoxin d, seg: staphylococcal enterotoxin g, seh: staphylococcal enterotoxin h, sei: staphylococcal enterotoxin l, sej: staphylococcal enterotoxin j, SCCmec: Staphylococcal casstte chromosmome mec, ST: sequence type, tpi: triosephosphate isomerase, yqi: acetyle coenzyme. respectively, were identical (Table 1), belonged to sequence type (ST) 764, clonal complex (CC) 5 [3] and carried SCCmec type II. These strains carried enterotoxins G and I, but no other SEs or TSST-1. Our patient was discharged uneventfully on day 156 after completing a two-month course of oral linezolide $(1200 \mathrm{mg} /$ day $)$ with intravenous clindamycin (CLDM; $1200 \mathrm{mg} /$ day) followed by teicoplanin $(400 \mathrm{mg} /$ day $)$ with intravenous CLDM (1200 mg/day) infusion.

\section{Discussion}

MRSA enterocolitis has been identified among Japanese patients since 1985, especially after undergoing gastric resection [4] or being medicated with a third-generation terocolitis have proceeded in Japan, MRSA is not a frequent cause of antibiotic-associated diarrhea and this notion has remained a matter of debate, as anecdotally reported elsewhere [6]. Therefore, to summarize and characterize published reports of MRSA enterocolitis in Japan, we intensively reviewed studies over the past dec(Table 2) [7-24]. We identified 36 (male, $\mathrm{n}=28$ female, $\mathrm{n}=8$ ) patients (age range, 0 to $91 \mathrm{y}$; mean $\pm \mathrm{SD}$; $59.5 \pm 21.3 \mathrm{y}$ ) who required a median of seven (range, 2-40) days of antibiotics to treat MRSA enterocolitis that was mainly diagnosed from stool cultures. Two were diagnosed at autopsy (patients 1 and 3 ) and one each was diagnosed by colonoscopy (patient 2) and during surgery (patient 4). Importantly, SEs or TSST-1 have been assessed in only four (11.1\%) patients (Nos. 1, 5, 6 and 8); two of them had enterotoxin $\mathrm{C}$ and all four had TSST-1. Furthermore, our review of two reports $[12,13]$ found that patients No. 5 and 6 (Table 2) satisfied the criteria for TSS with typical erythema and desquamation, but the authors had diagnosed them with MRSA Fith did not satisfy the criteria for TSS. None of the other patients with MRSA enterocolitis had cutaneous lesions similar to those of TSS. MRSA colitis clinically cephalosporin [5]. Although many studies of MRSA en- 
Table 2 Summary of methicillin-resistant Staphylococcus aureus enterocolitis reported in Japan over the past 10 years

\begin{tabular}{|c|c|c|c|c|c|c|c|c|c|}
\hline Pt. No. & Age & Sex & Underlying disease & Diagnostic method & $\begin{array}{c}\text { Site of } \\
\text { infection }\end{array}$ & $\begin{array}{l}\text { Duration* } \\
\text { (days) }\end{array}$ & $\begin{array}{c}\text { Enterotoxin/coagulase } \\
\text { Type/TSST-1 }\end{array}$ & Prog. & Ref \\
\hline 1 & 66 & M & Appendectomy & Autopsy & $\begin{array}{l}\text { All digestive } \\
\text { tract }\end{array}$ & NA & NA/II/+ & $\mathrm{D}$ & 9 \\
\hline 2 & 31 & M & Acute pancreatitis & $\begin{array}{c}\text { Stool culture with } \\
\text { Biopsy }\end{array}$ & Colon & 12 & NA & $\mathrm{D}$ & 10 \\
\hline 3 & 47 & $\mathrm{~F}$ & UTI & Autopsy & $\begin{array}{c}\text { Small } \\
\text { Intestine }\end{array}$ & 10 & NA & $\mathrm{D}$ & 7 \\
\hline 4 & 73 & M & Esophageal Ca, Gastric CA POS & Ileum interposition & none & 19 & NA & A & 11 \\
\hline 5 & 59 & M & Gastric Ca & Stool culture & NA & NA & $\mathrm{C} / \mathrm{NA}+$ & A & 12 \\
\hline 6 & 23 & M & Infected pilonidal sinus POS & NA & NA & 15 & $-/-/+$ & A & 13 \\
\hline 7 & 71 & M & $\begin{array}{l}\text { Peritonitis with mediastinitis } \\
\text { POS }\end{array}$ & NA & NA & 18 & NA & A & 14 \\
\hline 8 & 72 & M & Panperitonitis POS & Stool culture & NA & 3 & $\mathrm{~A} / \mathrm{II} /+$ & A & 15 \\
\hline 9 & 26 & $\mathrm{~F}$ & Tongue Ca POS & Stool culture & NA & 7 & NA & $\mathrm{D}$ & 16 \\
\hline 10 & 75 & $\mathrm{~F}$ & Gallbladder Ca & NA & NA & NA & NA & $\mathrm{D}$ & 8 \\
\hline 11 & 64 & M & Pancreatitis & Stool culture & NA & NA & NA & A & 17 \\
\hline 12 & 73 & $\mathrm{~F}$ & Pancreatitis & Stool culture & NA & NA & NA & A & 17 \\
\hline 13 & 44 & M & Pancreatitis & Stool culture & NA & NA & NA & $\mathrm{D}$ & 17 \\
\hline 14 & 77 & M & Pneumonia & Stool culture & NA & 42 & NA & A & 18 \\
\hline 15 & 78 & M & Ileus POS & Stool culture & NA & 20 & NA & A & 19 \\
\hline 16 & 78 & $\mathrm{~F}$ & Intestinal perforation POS & Stool culture & NA & NA & NA & $\mathrm{D}$ & 20 \\
\hline 17 & 39 & M & Acute mandibular periostitis & Stool culture & NA & 8 & NA & A & 21 \\
\hline 18 & 60 & M & $\begin{array}{l}\text { Lung Ca small intestine } \\
\text { metastasis POS }\end{array}$ & NA & NA & 7 & NA & A & 22 \\
\hline 19 & 84 & M & $\begin{array}{c}\text { Pneumatosis cystoides } \\
\text { intestinalis }\end{array}$ & Stool culture & NA & NA & NA & A & 23 \\
\hline 20 & 71 & M & $\mathrm{AMI}, \mathrm{CABG}$ & Stool culture & NA & 3 & NA & A & 24 \\
\hline 21 & 73 & M & Sigmoid colon Ca POS & Stool culture & NA & 2 & NA & A & \\
\hline 22 & 63 & M & Rectum Ca POS & Stool culture & NA & 2 & NA & A & \\
\hline 23 & 78 & M & UTI & Stool culture & NA & 4 & NA & A & \\
\hline 24 & 91 & $\mathrm{~F}$ & Pneumonia, cholecystitis & Stool culture & NA & NA & NA & A & \\
\hline 25 & 59 & M & Esophageal Ca POS & Fecal properties & NA & 4 & NA & A & \\
\hline 26 & 70 & $\mathrm{~F}$ & $\begin{array}{l}\text { Perforation of sigmoid colon } \\
\text { Ca POS }\end{array}$ & Stool culture & NA & 4 & NA & A & \\
\hline 27 & 64 & M & $\begin{array}{l}\text { Perforation of amebic colitis } \\
\text { POS }\end{array}$ & NA & NA & 4 & NA & A & \\
\hline 28 & 0 & M & BA & Stool culture & NA & 7 & NA & A & \\
\hline 29 & 18 & M & $\begin{array}{l}\text { Lymphoblastic lymphoma T- } \\
\text { cell type }\end{array}$ & Stool culture & NA & NA & NA & A & \\
\hline 30 & 64 & M & Renal abscess & NA & NA & NA & NA & A & \\
\hline 31 & 88 & $\mathrm{~F}$ & Pneumonia & NA & NA & 9 & NA & A & \\
\hline 32 & 64 & M & Pancreatic Ca POS & Stool culture & NA & NA & NA & A & \\
\hline 33 & 62 & M & Ileus & $\begin{array}{l}\text { Fluid drainage from } \\
\text { ileus tube }\end{array}$ & NA & 5 & NA & A & \\
\hline 34 & 66 & M & Esophageal Ca POS & NA & NA & NA & NA & A & \\
\hline 35 & 26 & M & ALL & Stool culture & NA & 14 & NA & $\mathrm{D}$ & \\
\hline 36 & 47 & $M$ & lleus & NA & NA & 9 & NA & $D$ & \\
\hline
\end{tabular}

A, alive; ALL, acute lymphocytic leukemia; AMI, acute myocardial infarction; BA, bronchial asthma; Ca, carcinoma; CABG, coronary artery bypass grafting; D, dead; Duration*, duration of preceding antibiotics therapy to diagnosis of MRSA enterocolitis; NA, not available; POS, postoperative status; Prog, prognosis; Pt. No, patient number; TSST-1, toxic shock syndrome toxin-1; UTI, urinary tract infection; All patients were described in Japanese and only patients 1 to 20 have been published in English and are cited as references. 
resembles TSS in many respects, such as shock, multiple organ failure and pyrexia due to MRSA bacteremia, but it should be differentiated from TSS regardless of the presence of TSST-1.

Although enterotoxins (A, A and B, D [6] or C $[25,26]$, and the bicomponent leukotoxin LukE-LukD [27]) have been detected in strains associated with MRSA enterocolitis, the relevance to this disease of enterotoxins $G$ and/or I that were recognized in our patient remains unknown. Humans are natural reservoirs of $S$. aureus, with intermittent colonization occurring in 30\% - 50\% of healthy adults [28], $10 \%$ of whom harbor this microorganism in the gastrointestinal tract [29]. The ratio of nasal MRSA carriage among healthy individuals in Japan is similarly high, at $38.0 \%$ [30]. In contrast, another report stated that most hospitalized geriatric patients carrying MRSA in the gastrointestinal space were treated with antibiotics [31]. These facts imply that most of the reported "MRSA enterocolitis" in Japan might have been caused by preceding antibiotic use and/or presented with one of the clinical features of superantigen-derived toxic shock syndrome due to TSST-1 or undetermined effects of SEs other than those described above. In addition, colonization of MRSA in the digestive tract after antibiotic use might have been misdiagnosed in some patients as a pathogen for diarrhea of undetermined causes.

Boyce et al. [6] reported that among five patients with MRSA enterocolitis in whom fecal leukocytes were examined, two were positive, like our patient. They also stated that the duration of preceding antimicrobial therapy ranged from 2 to 21 days, which is similar to that of other Japanese reports indicating a range of 2 to 42 days (Table 2). Boyce et al. [6] also reported that the maximum recorded stool volume per $24 \mathrm{~h}$ period ranged from 475 to $8,250 \mathrm{~mL}$ in 11 patients with MRSA enterocolitis and that the total volume of stool recorded for the entire diarrheal illness ranged from $745 \mathrm{~mL}$ to $38.85 \mathrm{~L}$, which was identical to that of our patient and other reports (maximum recorded stool volume per $24 \mathrm{~h}$ ranged from $500 \mathrm{~mL}$ to $4.0 \mathrm{~L}$ from patient Nos. 9, 15, 33 and the present patient). Froberg et al. [32] reported that MRSA enterocolitis features loosely adherent pseudomembranes in the small intestine, whereas $C$. difficile pseudomembranous colitis locates in the colon where pseudomembranes are tightly adherent. Indeed, the sites of inflammation in patients $1,2,3$ and 4 in our review comprised the gastric wall (patient 1 at autopsy), small intestine (patient 3 at autopsy; patient 4 by biopsy), or colon (patient 2, by biopsy), indicating that the preferred site of MRSA enterocolitis is the small intestine. Cultured samples from all four patients were positive for MRSA, but only patients 3 and 4 had white pseudomembranes at the small intestine that were MRSA- positive. Although pathological information is scant, MRSA enterocolitis can involve not only the small intestine, but also the colon (Table 2).

Colonic fiberscopy indicated that our patient had no apparent pseudomembranes up to the sigmoid portion. Nonetheless, MRSA enterocolitis was the most likely source of the massive diarrhea because $C$. difficile toxin A/B was negative, MRSA was the dominant organism isolated from abundant fecal leukocytes, the watery diarrhea was refractory to metronidazole but susceptible to oral VCM and suppurative MRSA arthritis of the right hip joint occurred after the watery diarrhea or transient MRSA bacteremia were identified. Watanabe et al. [33] found identical $(69.2 \%)$ or similar (23.1\%) PFGE profiles in 12 of 13 samples obtained from the respiratory tract (nasal cavity and/or pharynx and/or sputum) and stool samples from patients infected with MRSA. They speculated that one cause of enterocolitis could be MRSA that originated from colonization of the respiratory tract and was absorbed into the digestive tract. A single genetic event in MRSA strains colonizing the respiratory tract might generate a new MRSA strain that could cause enterocolitis, septicemia and septic arthritis. As the toxin gene profiles among strains from blood, synovial fluid and sputum were identical on MLST (SCCmec type II, ST764, CC5), whether or not a single minor genetic change could alter virulence remains uncertain. Further studies are required to clarify the reason for the change in virulence.

The MRSA strain derived from stool cultures did not thrive. Thus, we could not perform MLST after obtaining convincing PFGE results. Nevertheless, we postulate that our patient had MRSA enterocolitis that was sequentially complicated with septic arthritis. The relationship between MRSA enterocolitis and SEs or TSST-1 together with pathological assessments that could improve understanding of the pathological process involved in MRSA enterocolitis require further exploration.

\section{Conclusion}

Any MRSA isolated from patients with diarrhea that is refractory to treatment should be assessed from a multidisciplinary viewpoint considering colonization or pathogens. Our review of published reports suggests that the clinical features MRSA enterocolitis mimic those of TSS, which might reflect undetermined effects of SEs or TSST-1 associated with MRSA infection.

\section{Consent}

Written informed consent was obtained from the patient for publication of this Case Report and any accompanying images. A copy of the written consent is available for review by the Editor-in-Chief of this journal. 


\section{Abbreviations}

CC: Clonal complex; CLDM: Clindamycin; Luk: Leukocidin; MLST: Multilocus sequence typing; MRI: Magnetic resonance imaging; MRSA: Methicillinresistant Staphylococcus aureus; PFGE: Pulsed-field gel electrophoresis; SEs: Staphylococcal enterotoxins; ST: Sequence typing; TSST-1: Toxic shock syndrome toxin-1; VCM: Vancomycin.

\section{Competing interests}

The authors declare that they have no competing interests.

\section{Authors' contributions}

YO, TS, TK, and KO mainly managed the patient. YO, TS, HT, and HG wrote the manuscript. KK, KA, SY, and HM performed microbiological analyses. All authors read and approved the final manuscript.

\section{Author details}

'Department of Respiratory Medicine, Kyorin University School of Medicine, 6-20-2 Shinkawa, Mitaka, Tokyo 181-8611, Japan. 'Department of Infection Control Science, Faculty of Medicine, Juntendo University, Tokyo, Japan. ${ }^{3}$ Department of Clinical Laboratory, Kyorin University School of Medicine, Tokyo, Japan.

Received: 18 September 2013 Accepted: 3 January 2014

Published: 9 January 2014

\section{References}

1. Tenover FC, Arbeit RD, Goering RV, Mickelsen PA, Murray BE, Persing DH Swaminathan B: Interpreting chromosomal DNA restriction patterns produced by pulsed-field gel electrophoresis: criteria for bacterial strain typing. J Clin Microbiol 1995, 33(9):2233-2239.

2. Case definitions for infectious conditions under public health surveillance. Centers for disease control and prevention. MMWR Recomm Rep 1997, 46(RR-10):1-55. http://www.ncbi.n/m.nih.gov/pubmed/9148133.

3. Multi Locus Sequence Typing home page. http://www.mlst.net/

4. Morita H, Tani M, Adachi H, Kawai S: Methicillin-resistant Staphylococcus aureus (MRSA) enteritis associated with prophylactic cephalosporin administration and hypochlorhydria, after subtotal gastrectomy. Am J Gastroenterol 1991, 86(6):791-792.

5. Hori K, Yura J, Shinagawa N, Sakurai S, Mashita K, Mizuno A: Postoperative enterocolitis and current status of MRSA enterocolitis-the result of a questionnaire survey in Japan. Kansenshogaku Zasshi 1989, 63(7):701-707.

6. Boyce JM, Havill NL: Nosocomial antibiotic-associated diarrhea associated with enterotoxin-producing strains of methicillin-resistant Staphylococcus aureus. Am J Gastroentero/ 2005, 100(8):1828-1834.

7. Fujita K, Honda Y, Kurihara T, Ohba H, Okimoto N: A case of pseudomembranous enterocolitis caused by methicillin-resistant Staphylococcus aureus. J J A Inf D 2004, 78:905-909.

8. Ohkouchi S, Gomi K, Tokue Y, Kikuchi T, Fujimura S, Nukiwa T, Watanabe A: Sepsis caused by methicillin-resistant Staphylococcus aureus (MRSA) without effects of vancomycin (VCM), teicoplanin (TEIC) and arbekacin (ABK) treated successfully and safely with intravenous administration of linezolid (LZD). Jpn J Chemother 2004, 52(12):787-792.

9. Tei M, Shimizu J, Ikeda K, Kitada M, Shimano T: Whole digestive tract infection caused by methicillin-resistant Staphylococcus aureus after appendectomy. J Jpn Surg Assoc 2006, 67(8):1739-1743.

10. Hamada $Y$, Imaizumi H, Watanabe M, Kikuchi H, Watanabe M, Nishimaki H, Kida M, Sunakawa K, Souma K, Saigenji K, Matsubara H, Yago K: Continuous regional arterial infusion therapy with biapenem and nafamostat mesilate for severe acute pancreatitis. J Jpn Pancreas Sci 2008, 23(5):578-586.

11. Kitahara $Y$, Nakamura M, Iwahashi M, Nakamori M, Ojima T, Yamaue H: A case of pseudomembranous enterocolitis caused by methicillin-resistant Staphylococcus aureus in the interposed ileum after esophagectomy. J Jpn Surg Assoc 2011, 72(10):2524-2529.

12. Shimizu T: A case of toxic shock syndrome(TSS) induced by MRSA in post operation period of gastric cancer. Niigata lgakkai Zasshi 2009, 123(4):181-187.

13. Hayashi T, Yoshizawa M, Watanabe N, Murayama Y: Two cases of toxic shock syndrome treated by blood purification therapy. J Jpn Sci Surg Infect 2006, 3(2):167-170.

14. Higuchi R, Ota T, Kajiyama H, Yazawa T, Uemura S, Sato T, Nakamura T, Yamamoto M: A case of surgical drainage in post coronary artery bypass grafting complicated with diffusing peritonitis and mediastinitis. J Abdom Emerg Med 2011, 31(3):561-565.

15. Higuchi T, Sato N, Otsuka K, Ito N, Kawasaki Y, Fujiwara H, Kashiwada T, Hayakawa Y, Asahi H, Saito K, Sato S: A case of MRSA enteritis with idiopathic perforation of the sigmoid colon. J Jpn Sci Surg Infect 2002, 14:45-50.

16. Yanagi Y, Kubota T, Matsui K, Kadokura Y, Hanaoka H: Enterocolitis caused by methicillin-resistant Staphylococcus aureus (MRSA) after bilateral neck dissection. Practica Otologica 2002, 95(4):409-412.

17. Mizumoto S, Hitani Y, Akashi H, Sasaki M, Kurokawa E, Yamamoto H, Okano K, Taniguchi H, Takami K, Umeshita K: A case of gallbladder cancer with duplication of common bile duct and anomalous junction of pancreaticobiliary duct. Jpn J Gastroenterol Surg 2003, 36(5):378-382.

18. Uchida E, Furukawa K, Matsushita A, Aimoto T, Nakamura Y, Katsuno A, Chou K, Tajiri T: The significance of intestine and bacterial translocation in severe acute pancreatitis. The Journal of the Japanese Society of Clinical Studies on Intestinal Microflora 2004, 1:17-21.

19. Hatakeyama J, Inomata $\mathrm{H}$, Sato N: A case of MRSA enterocolitis occurred after ileus operation. J Jpn Coll Surg 2005, 30(6):882-884.

20. Hashimoto $Y$, Sakashita $Y$, Takamura M, Iwako H, Watanabe $Y$, Shigemoto N, Kin K: Massive rectroperitoneal emphysema caused by the stercoral perforation of the sigmoid colon. Jpn J Gastroentero/ Surg 2005, 38(5):566-570.

21. Nishizawa K, Terasaka S, Hirai E, Kuga Y: A case of shock and DIC because of MRSA enteritis during the medical treatment of odontogenic infection. Kyüshū Shika Gakkai Zasshi 2005, 59(1):22-26.

22. Miyake T, Shimizu T, Sonoda H, Mekata E, Murata S, Tani T: A case of intestinal metastasis of lung cancer undedicated preoperative diagnosis. J Jpn Coll Surg 2011, 36(5):787-791.

23. Fujii $Y$, Takagi l: A case of pneumotosis cytoides intestinalis associated with MRSA enteritis. J Jpn Coll Surg 2010, 71(12):3158-3161.

24. Nakai $Y$, Nakata $\mathrm{T}$, Sato $\mathrm{H}$ : A case of enterocolitis caused by methicillinresistant Staphylococcus aureus after coronary artery bypass grafting. J Clin Med Imabari Med Assoc 2007, 20(1):22-26.

25. Takesue $Y$, Yokoyama T, Kodama T, Santou T, Nakamitsu A, Murakami Y, Imamura $Y$, Miyamoto $\mathrm{K}$, Okita M, Tsumura H: Toxin involvement in methicillin-resistant Staphylococcus aureus enteritis in gastroenterological surgery. Gastroenterol Jpn 1991, 26(6):716-720.

26. Kodama T, Santo T, Yokoyama T, Takesue Y, Hiyama E, Imamura Y, Murakami Y, Tsumura H, Shinbara K, Tatsumoto N, Matsuura Y: Postoperative enteritis caused by methicillin-resistant Staphylococcus aureus. Surg Today 1997, 27(9):816-825

27. Gravet A, Rondeau M, Harf-Monteil C, Grunenberger F, Monteil H, Scheftel JM, Prevost G: Predominant Staphylococcus aureus isolated from antibiotic-associated diarrhea is clinically relevant and produces enterotoxin a and the bicomponent toxin LukE-lukD. J Clin Microbio 1999, 37(12):4012-4019.

28. Casewell MW, Hill RL: The carrier state: methicillin-resistant Staphylococcus aureus. J Antimicrob Chemother 1986, 18(Suppl A):1-12.

29. Christie CD, Lynch-Ballard E, Andiman WA: Staphylococcal enterocolitis revisited: cytotoxic properties of Staphylococcus aureus from a neonate with enterocolitis. Pediatr Infect Dis J 1988, 7(11):791-795.

30. Komori Y: Epidemic Study of Nasal Carriage of Methicillin-Resistant Staphylococci (MRSA and MRC-NS) in the Volunteers without Apparent Infectious Disease. http://kaken.nii.ac.jp/d/p/14570308.ja.html.

31. Masaki H, Yoshimine H, Miyata K, Nakanishi T, Degawa S, Takahashi H, Kuroki R, Morimoto K, Kaida S, Matsumoto K: a clinical study of gastrointestinal flora in patients with, or without MRSA colonization in the upper-respiratory tract after introduction of preventive measures of hospital infection. Kansenshogaku Zasshi 1995, 69(11):1260-1268.

32. Froberg MK, Palavecino E, Dykoski $R$, Gerding DN, Peterson LR, Johnson S: Staphylococcus aureus and clostridium difficile cause distinct pseudomembranous intestinal diseases. Clin Infect Dis 2004, 39(5):747-750.

33. Watanabe H, Masaki H, Asoh N, Watanabe K, Oishi K, Kobayashi S, Sato A Nagatake T: Enterocolitis caused by methicillin-resistant Staphylococcus aureus: molecular characterization of respiratory and digestive tract isolates. Microbiol Immunol 2001, 45(9):629-634.

\section{doi:10.1186/1756-0500-7-21}

Cite this article as: Ogawa et al:: Methicillin-resistant Staphylococcus aureus enterocolitis sequentially complicated with septic arthritis: a case report and review of the literature. BMC Research Notes 2014 7:21. 